\title{
LETTER
}

\section{Standardized liberation trials in patients with COVID-19 ARDS treated with venovenous extracorporeal membrane oxygenation: when ready, let them breathe!}

\author{
Ricardo Teijeiro-Paradis ${ }^{1}$, Pradip Tiwari ${ }^{2}$, Amanda Spriel ${ }^{3}$, Lorenzo Del Sorbo ${ }^{1}$ and Eddy Fan ${ }^{1,4,5^{*}}$ (I)
}

(๑) 2021 Springer-Verlag GmbH Germany, part of Springer Nature

\section{Dear Editor,}

Venovenous (VV) extracorporeal membrane oxygenation (ECMO) has proven to be useful in the management of patients with severe acute respiratory distress syndrome (ARDS) associated with coronavirus disease 2019 (COVID-19) [1]. Due to its invasive nature, timely liberation from ECMO should become a priority when it is no longer needed for gas exchange or to attenuate ventilator induced lung injury. Current liberation strategies are variable [2-4] and may lead to both under-recognition of readiness or unsafe initiation of liberation attempts.

We designed a single-center, retrospective cohort study of adult patients treated with VV ECMO for COVID-19 ARDS at Toronto General Hospital between May and October 2020. The objective of the study was to assess the feasibility of adopting a standardized liberation protocol. We introduced an alternative approach to liberation, incorporating a daily assessment of eligibility for ECMO liberation trials. Entry criteria included $12 \mathrm{~h}$ of treatment for ARDS, no neuromuscular blockade, hemodynamic stability, reasonable ventilation parameters, and extracorporeal blood flow $\leq 5 \mathrm{~L} / \mathrm{min}$ and sweep gas flow $(\mathrm{SGF}) \leq 4$ L/min (Supplementary material Table 1). Readiness for liberation was assessed by conducting standardized liberation trials (SLTs) (Fig. 1-Panel A and supplementary material Tables 2 and 3). SLTs consisted in interrupting SGF (i.e., 4-0 L/min), emulating spontaneous breathing

\footnotetext{
*Correspondence: eddy.fan@uhn.ca

${ }^{1}$ Interdepartmental Division of Critical Care Medicine, University of Toronto, Toronto, Canada

Full author information is available at the end of the article
}

trials. For successful SLTs, patients would be maintained off SGF until decannulation (usually within $24 \mathrm{~h}$ ). In failed SLTs, patients would continue to be trialed daily. Differences in ventilatory, hemodynamic and blood gas parameters were collected and compared between SLT outcomes using Mann-Whitney $U$ test.

We performed 61 SLTs in 31 patients, with 19 SLTs (31\%) leading to decannulation (Supplementary material Table 4-Fig. 1). Seventy-three percent of decannulated patients were liberated from higher SGF than usual practice (SGF $\geq 2 \mathrm{~L} / \mathrm{min}$ ), with $63 \%$ requiring $\leq 2$ SLTs (Fig. 1-Panel B). At trial termination, failed SLTs had different respiratory mechanics, ventilatory ratio and worse oxygenation (Fig. 1-Panel $\mathrm{C}$ and supplementary material Table 5). Median time to SLT failure was $0.25 \mathrm{~h}$ (IQR 0.25-1.5). The most common reasons for failing were hypoxemia $(40 \%)$ and increased work of breathing (36\%).

The rate of SLT related complications was 3\% (respiratory acidosis and atrial fibrillation). Two patients (10\%) failed decannulation [5]. One patient was recannulated and ultimately decannulated after treating for a new ventilator associated pneumonia. Four patients (23\%) died after decannulation (two died of non-respiratory causes, one died of unrelated pulmonary hemorrhage, one died of respiratory failure and sepsis [decannulation failure]). Median time to death was 7.5 days (IQR 6-13) (Supplementary material Figs. 3 and 4). All remaining decannulated patients were subsequently extubated (median 11 days, IQR 2-25). 


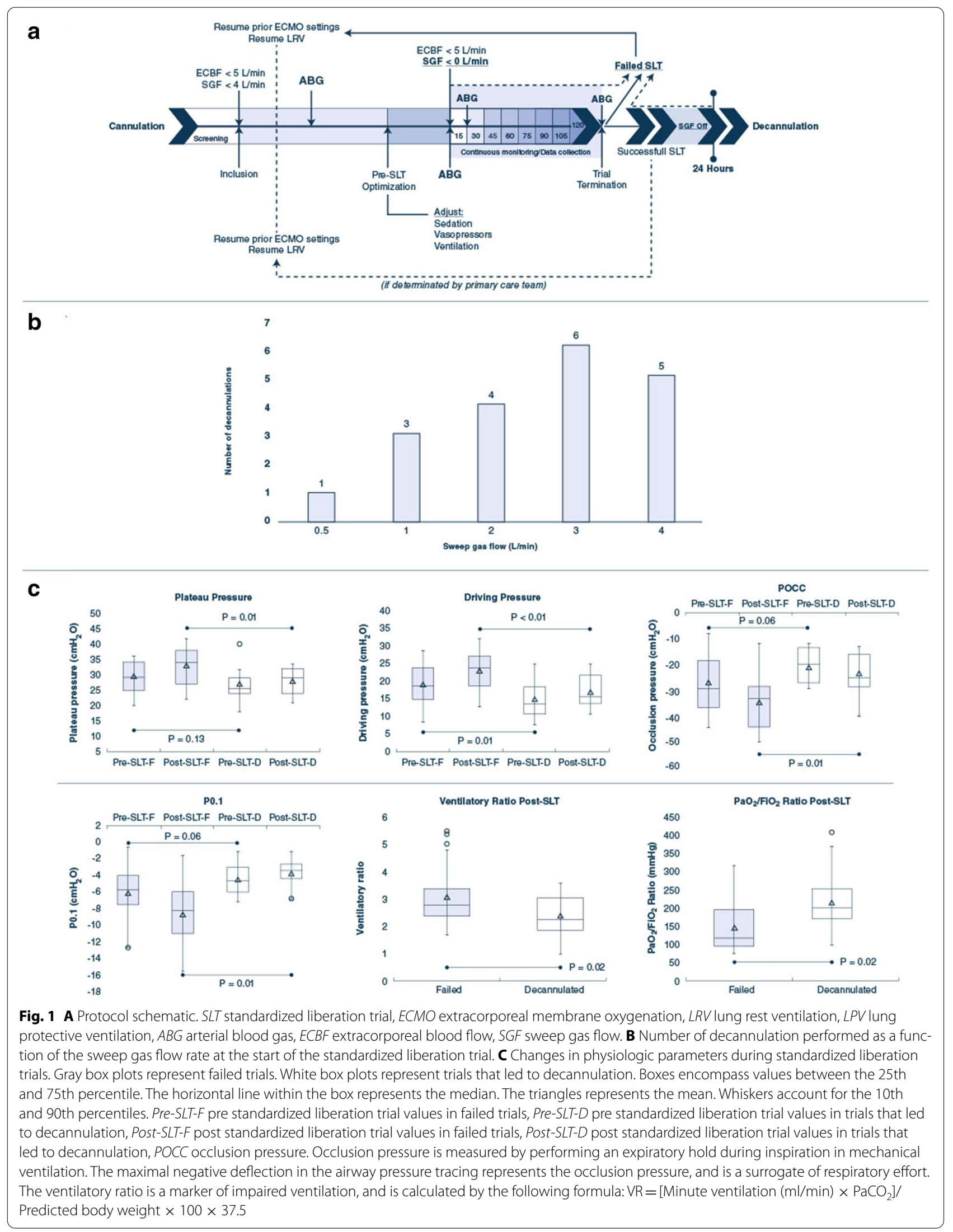


We developed and tested a standardized liberation protocol incorporating defined entry criteria and pragmatic liberation trials to identify patients with enough lung recovery to be liberated from ECMO. Given the small sample size and exploratory nature of our study, we did not explore the utility of collected variables for predicting successful decannulation. Although hypothesis generating, early identification of patients ready to be liberated may potentially allow for shorter duration of support, reducing ECMO related costs and complications. The limitations of the study include its retrospective observational nature, small population and single center distribution. We did not capture duration of sedation or paralysis, however, all patients needed to be off paralysis for at least $24 \mathrm{~h}$ prior to a SLT. As no universal definition of successful decannulation exists, we were unable to fully explore the safety of incorporating SLTs to clinical practice. Further research into liberation from ECMO is needed. Extrapolating from research on liberation from mechanical ventilation, where a systematic assessment demonstrated superiority to clinical judgement and preference, it is intuitive to think that protocolized approaches to liberation form ECMO will follow a similar path.

Supplementary Information

The online version contains supplementary material available at https://doi. org/10.1007/s00134-021-06523-X.

\section{Author details \\ ${ }^{1}$ Interdepartmental Division of Critical Care Medicine, University of Toronto, Toronto, Canada. ${ }^{2}$ Civil Service Hospital, Kathmandu, Nepal. ${ }^{3}$ Perfusion Department, University Health Network, Toronto, Canada. ${ }^{4}$ Institute of Health Policy, Management and Evaluation, University of Toronto, Toronto, Canada. \\ ${ }^{5}$ Toronto General Hospital, 585 University Avenue, PMB 11-123, Toronto, ON M5G 2N2, Canada.}

\section{Author contributions}

RT-P: conceptualization, methodology, data collection, analysis, manuscript preparation. PT: conceptualization, data collection, review and editing. AS: conceptualization, methodology, data storage, review and editing. LDS: conceptualization, methodology, review and editing. EF: conceptualization, methodology, analysis, review and editing.

\section{Funding}

Not applicable.
Availability of data and material

Not applicable.

Code availability

Not applicable.

Declarations

Conflicts of interest

EF reports personal fees from ALung Technologies, Baxter, Boehringer-Ingelheim, GE Healthcare, and MC3 Cardiopulmonary outside the submitted work. No conflicts of interest/competing interests for all other remaining authors.

\section{Ethics approval}

This retrospective chart review study involving human participants was in accordance with the ethical standards of the institutional and national research committee and with the 1964 Helsinki Declaration and its later amendments or comparable ethical standards. The Human Investigation Committee (IRB) of University Health Network (REB 20-6142) approved this study.

\section{Consent to participate}

Not applicable.

\section{Consent for publication}

Not applicable.

\section{Publisher's Note}

Springer Nature remains neutral with regard to jurisdictional claims in published maps and institutional affiliations.

Received: 1 July 2021 Accepted: 30 August 2021

Published online: 16 September 2021

References

1. Shaefi S, Brenner SK, Gupta S, O'Gara BP, Krajewski ML, Charytan DM et al (2021) Extracorporeal membrane oxygenation in patients with severe respiratory failure from COVID-19. Intensive Care Med 47(2):208-221

2. Grant AA, Hart VJ, Lineen EB, Badiye A, Byers PM, Patel A et al (2018) A weaning protocol for venovenous extracorporeal membrane oxygenation with a review of the literature. Artif Organs 42(6):605-610

3. Vasques F, Romitti F, Gattinoni L, Camporota L (2019) How I wean patients from veno-venous extra-corporeal membrane oxygenation. Crit Care 23(1):316

4. Broman LM, Malfertheiner MV, Montisci A, Pappalardo F (2018) Weaning from veno-venous extracorporeal membrane oxygenation: how I do it. J Thorac Dis 10(Suppl 5):S692-S697

5. Al-Fares AA, Ferguson ND, Ma J, Cypel M, Keshavjee S, Fan E et al (2021) Achieving safe liberation during weaning from $\mathrm{W}$-ECMO in patients with severe ARDS: the role of tidal volume and inspiratory effort. Chest. https://doi.org/10.1016/j.chest.2021.05.068 